\title{
An Electronic Circuit Model of the Interpostsynaptic Functional LINK Designed to Study the Formation of Internal Sensations in the Nervous System
}

\author{
Kunjumon I. Vadakkan ${ }^{1,2}$ \\ ${ }^{1}$ Division of Neurology, Department of Internal Medicine, Faculty of Medicine, University of Manitoba, \\ GF532-820 Sherbrook Street, Winnipeg, MB, Canada R3A 1R9 \\ ${ }^{2}$ Division of Neurology, Department of Medicine, University of Toronto, Sunnybrook Health Sciences Centre, \\ 2075 Bayview Avenue, Room A4-08, Toronto, ON, Canada M4N 3M5
}

Correspondence should be addressed to Kunjumon I. Vadakkan; kunjumon.vadakkan@utoronto.ca

Received 5 August 2014; Accepted 12 October 2014; Published 3 December 2014

Academic Editor: Shuai Li

Copyright (C) 2014 Kunjumon I. Vadakkan. This is an open access article distributed under the Creative Commons Attribution License, which permits unrestricted use, distribution, and reproduction in any medium, provided the original work is properly cited.

\begin{abstract}
The nervous system makes changes in response to the continuous arrival of associative learning stimuli from the environment and executes behavioral motor activities after making predictions based on past experience. The system exhibits dynamic plasticity changes that involve the formation of the first-person internal sensations of perception, memory, and consciousness to which only the owner of the nervous system has access. These properties of natural intelligence need to be verified for their mechanism of formation using engineered systems so that a third person can access them. In the presence of a synaptic junctional delay of up to two milliseconds, we anticipate that the systems property of formation of internal sensations is likely independent of the mode of conduction along the neuronal processes. This allows testing for the formation of internal sensations using electronic circuits. The present work describes the neurobiological context for the formation of the basic units of inner sensations that occur through the reactivation of interpostsynaptic functional LINKs and its connection to motor activity. These mechanisms are translated to an analogue circuit unit for the development of robotic systems.
\end{abstract}

\section{Introduction}

Realistic models of the nervous system are based on known features of neurons and synapses and have been integrated into microcircuits $([1,2]$ reviews $[3,4])$. What additional features are needed in the transfer of natural intelligence to artificial intelligence? Nervous system combines newly arriving sensory information with that of the internal sensations of retrieved memories of the past experience to make predictions. These inner sensations guide behavioral motor actions that enable survival of the animal in the physical world. The formation of inner sensations towards which only the owner of the nervous system has access is the basis of natural intelligence. Since it is not possible to study the firstperson-accessible inner sensations in biological systems, it is required to replicate the mechanism in engineered systems possibly by using silicon-based electronics. Even though some of the earlier studies were focused on replicating the nervous system's first-person properties [5], a direct approach to test theoretically feasible mechanisms has not been carried out. In order to understand the functional emergence of the mind and its operations, it is necessary to make reasonable assumptions to fill the gaps in the experimental evidence [6] and test for novel methods to approach the first-person inner sensations. The surrogate markers in response to the internal sensations formed in the mind such as perception, memory, and consciousness are behavioral motor activities. In this regard, robotics is ideal to replicate the nervous system functions where the units that produce basic units of internal sensations can be operated to exhibit their integrated 
product of internal sensations that can be made to express surrogate motor outputs. The first-person feature of intrinsic motivation engaging in one's own sake out of curiosity and exploration was examined and modeled [7]. This was followed by development of computational reinforcement learning frameworks to encompass the autonomous development of skill hierarchies [8]. Further advancement in this direction is models for developmental robotics that explores potential cognitive processes underlying intrinsic motivation [9].

Reverse engineering the nervous system requires utilizing the empirical evidence from cellular, network, and systems level to build hypotheses about how neurobiological systems use sensory information to learn and act intelligently. Using the third-person observed findings from different faculties of brain sciences such as electrophysiology, neurobiology, systems neuroscience, behavioral neuroscience, perception science, and consciousness studies, it is possible to synthesize hypotheses that can explain brain functions from a first-person frame of reference. Cellular changes during associative learning between sensory inputs of vision (light), hearing (sound), smell, taste, and touch are expected to cause changes such that at a later point of time the fastest arriving stimulus among the associatively learned stimuli can evoke the internal sensation of late-arriving or nonarriving sensations. This permits rapid assessment of the environment by the nervous system for appropriate behavioral motor responses. A universal framework is required to explain how the system can remain in a conscious state to perceive sensory information from the environment and respond to this information through motor activities based on prior learning experience. Once a hypothesis that can explain almost all the brain functions is derived, it can be followed by designing experiments to verify the formation of the first-person properties that can be accessed by a third person. A general outline of a hypothesis in this direction was made previously [10].

The specific mechanism of the formation of internal sensations should be capable of operating in unison with the known synaptic connections between the neurons. This mechanism must explain memory retrieval at physiological time-scales, changes in the formation of internal sensations with changes in the frequency of oscillating neuronal activity at certain neuronal orders (e.g., the induction of sleep), the observed relationship between memory and consciousness [11-18], and the relationship between long-term potentiation (LTP) and behavioral motor activity that are currently used as surrogate markers for the formation of internal sensations of retrieved memories. In short, any mechanism involving the firing of neurons including those by the motor neurons that results in behavioral motor activity that are examined by third persons must explain how it is related to the formation of the internal sensation of higher brain functions and how it enables the system to plan and execute motor activity. With the aim to meet these criteria, the semblance hypothesis was developed $[19,20]$.

\section{Solving for the First-Person Properties}

Perception, memory, and consciousness consist of the formation of internal sensations of different complexities. The formation of the latter depends on the maintenance of an oscillatory pattern of neuronal activity at certain specific frequencies. Oscillating pattern of neuronal activities recorded using surface electroencephalogram has shown that synchronous neural oscillations are associated with cognitive processes such as memory, attention, and consciousness and in diseases of the mind [21] reviews [22, 23]. A decrease in alpha power has been linked to increasing demands of alertness and attention [24]. Theta power was shown to increase during encoding $[24,25]$ and theta and gamma oscillations are linked to working memory [26]. Changing their frequency during both sleep and anesthesia $[27,28]$ alters the formation of normal internal sensations. Synaptic transmission occurring at the synapses between the vertically arranged orders of neurons within the cortices can contribute towards the vertical component for the oscillatory pattern of firing of neurons. The horizontal component that leads to the waveform shape of the neuronal firing requires definite mechanisms. Even though gap junction proteins were thought to contribute to the lateral spread of activity, the persistence of high frequency $(150 \mathrm{~Hz})$ rhythms in connexin 36 -deficient mice $[29,30]$ requires an additional mechanism to explain. Even though the presence of gap junctions between excitatory neurons was searched for, convincing evidence for their presence was not available [31]. The semblance hypothesis has proposed interpostsynaptic functional LINKs (letters are capitalized only to show its significance) as a mechanism that can provide a sufficient horizontal component at locations of convergence of inputs, some of such locations where oscillating neuronal activity is observed.

Synaptic transmission takes place unidirectionally. Synaptic vesicles in the presynaptic terminals release their contents continuously, even at rest, into the synaptic cleft (quantal release) that contributes to the miniature excitatory postsynaptic potential (mEPSP) recorded from the neuronal soma (Figure 1(a)). Activity (action potential) arriving at the excitatory presynaptic terminal leads to the generation of excitatory postsynaptic potential (EPSP) (Figure 1(b)). Continuous unidirectional neurotransmission in the form of quantal release at the synapse activating the postsynaptic terminal receptors provides the context that incidental activation of the postsynaptic terminal by artificial means can induce the cellular hallucination (semblance formation) of an activity arriving from its presynaptic terminal (Figure 2). Can the cellular hallucination occurring by activation of the postsynapse be expressed in terms of sensory stimuli? The sensory meaning imparted to the system by the cellular hallucination can be derived by extrapolating the activity from the presynaptic terminal towards the sensory receptors. The derived packet of sensory stimuli constitutes the units of virtual internal sensations (Figure 2). The artificial stimulation of sensory cortices that induce hallucinations of respective sensory modalities [32] is likely to operate by this mechanism. 

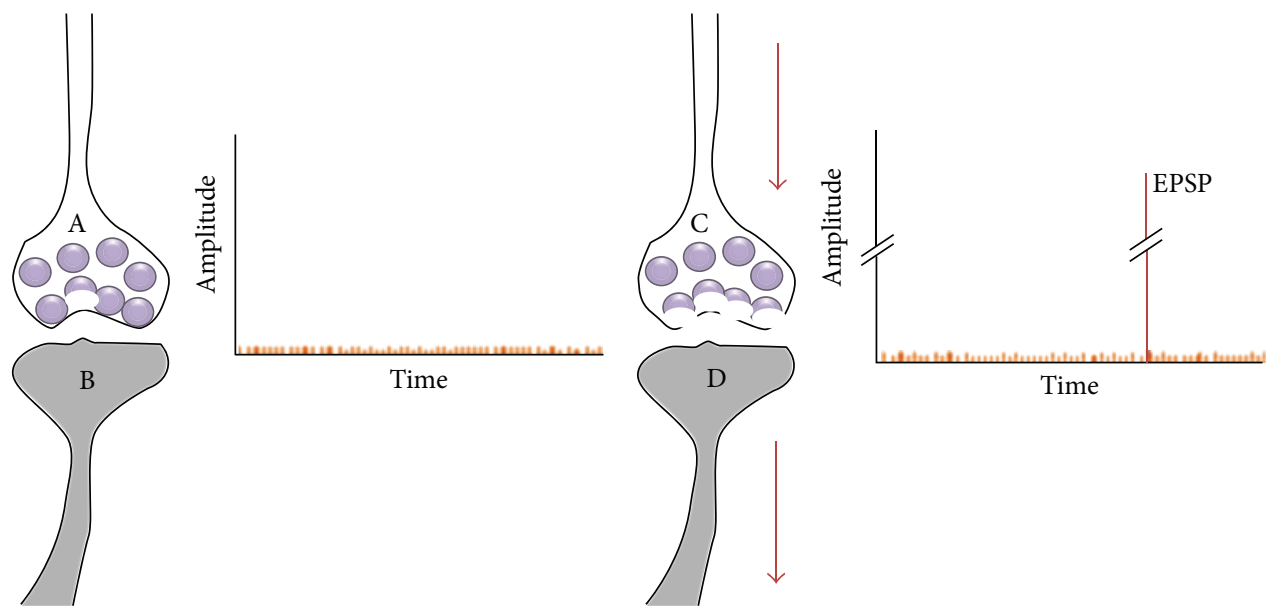

(a)

(b)

FIGURE 1: Diagrammatic representation of a synapse at rest and during arrival of an activity. (a) Synapse [A-B] at rest showing the quantal release of neurotransmitters from the presynaptic terminal A resulting in the formation of miniature potentials at the postsynaptic membrane B. The fact that it is difficult to block mEPSP recorded from the neuronal cell body even in experimental conditions indicates that it is likely an evolutionarily highly conserved mechanism. On the right side of the synapse is a graph showing miniature potentials at the postsynapse $\mathrm{B}$ at rest (axes are not scaled). (b) A second synapse [C-D] where the arrival of activity at the presynaptic terminal C results in the release of large number of synaptic vesicles resulting in the generation of EPSP at the postsynaptic terminal D. On the right side of the synapse is a graph showing potentials at the postsynapse D at rest (baseline) and during artificial stimulation (one spike) (they are not scaled and only intended to show the difference).

The mechanism of semblance formation can support the observations that artificial stimulation of visual cortex induces visual hallucinations $[34,35]$. Since visual hallucinations take place only when neuronal activity at certain neuronal orders shows an oscillatory pattern at certain frequencies [36], the cellular hallucination at the postsynapses is expected to form as a property of such systems. In a similar way, in order for internal sensations to occur in the form of semblances during higher brain functions such as perception, memory, and consciousness, it is necessary to have a mechanism to activate the postsynaptic terminal from its extrasynaptic locations. Such lateral activation can occur if the specific postsynapse where the unit of cellular hallucination is expected to be generated is LINKed to a second postsynapse. If such interpostsynaptic functional LINKs (Figure 3 ) are formed as a function of simultaneous activation of two abutted postsynaptic terminal membranes during associative learning, then their reactivation by the cue stimulus can induce semblances responsible for internal sensation of retrieved memories (Figure 4).

What is the likely nature of the LINK between the postsynapses? Postsynaptic membrane hemifusion [20], ephaptic transmission between the postsynaptic terminals, and a mechanism operating through extracellular matrix space are the possible candidate mechanisms. The duration for which the functional LINK is maintained can determine the duration for which it can be reactivated to induce the retrieval of memory. This can explain different types of memories based on their duration. Once newly formed, the LINK is reversible and can explain working memory. Repetitive activation or reactivation of the interpostsynaptic functional LINK by repetition of learning or related learning can result in its stabilization and can explain long-term memory. In addition to the formation of semblance, the activity arriving at the LINKed postsynapse can propagate further downstream and can lead to the firing of subthreshold activated neurons. If these neurons are motor neurons, then it can explain the initiation of spontaneous behavioral motor activity. Both innate and acquired interpostsynaptic functional LINKs are expected to carry out other higher brain functions.

The duration of time before the interpostsynaptic functional LINK reverses back to its original state determines halflife of the LINK. For example, if the mechanism operates through reversible interpostsynaptic membrane hemifusion, then insertion of transmembrane protein across the hemifused area can stabilize it until the life of the protein is over. The exact nature of the functional LINK is not known. Even though the presence of stabilized membrane hemifusion can be observed in electron microscopic pictures (Figures 2 and 4 in [37]), reversible nature of newly formed hemifused areas that can take place at very small percentage of the total area of the dendritic spines requires new technologies to track their changes in real time. Additional mechanisms for the interpostsynaptic functional LINKs are also possible including ephaptic transmission [38]. Even though structural evidence can be examined in biological systems, it will not be possible to demonstrate the first-person nature of the semblances within the biological systems. For this reason, it is necessary to replicate the mechanism in physical systems and explore ways to test for its formation.

Replicating the mechanism in a physical system is expected to be successful due to the following reasons. Nerve conduction takes place through the spread of depolarization along neuronal membranes. At the junctions between 


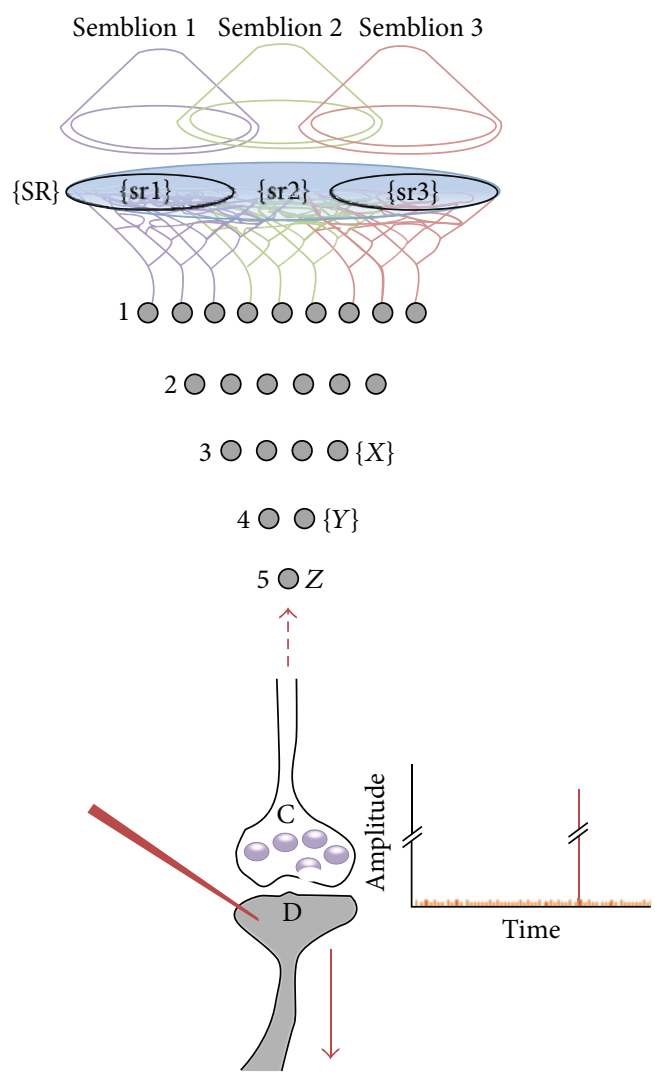

FIgURE 2: Artificial stimulation of a postsynapse is expected to induce the semblance of the arrival of activity from the presynaptic terminal as a systems property. This is exemplified by the induction of visual hallucinations of a simple or complex nature depending on the location of stimulation along the visual pathway in response to either artificial or pathological conditions [32]. In the figure, it is shown that the stimulation of postsynapse $\mathrm{D}$ evokes the cellular illusion of an activity reaching presynapse C. What sensory meaning does stimulation of the postsynapse impart to the system? It is necessary to search for the virtual inner sensation formed at postsynapse D. This can be estimated as the following. Sensory identity of the semblance of activity occurring at postsynapse D consists of inputs from the set of neurons $\{Y\}$ that synapse to neuron $Z$. The set of neurons $\{Y\}$ is normally activated by inputs from a set of lower-order neurons $\{X\}$. The set of neurons $\{X\}$ in turn is activated by another set of its lower-order neurons. Continuing this extrapolation towards the sensory level can identify a set of sensory receptors $\{\mathrm{SR}\}$. The semblance of activity induced by stimulating postsynapse $\mathrm{D}$ is that of a stimulus activating the sensory receptor set $\{S R\}$. Is it necessary to activate the whole receptor set $\{S R\}$ to activate postsynapse $\mathrm{D}$ ? It may become possible to activate postsynapse $\mathrm{D}$ by activating one of the receptor subsets $\{\mathrm{sr} 1\},\{\mathrm{sr} 2\}$, or $\{\mathrm{sr} 3\}$ of the receptor set $\{\mathrm{SR}\}$. The hypothetical packets of sensory stimuli capable of activating the sensory receptor sets $\{\operatorname{srl}\},\{\operatorname{sr} 2\}$, and $\{\mathrm{sr} 3\}$ are called semblions 1,2 , and 3 , respectively (modified from [33]). On the right side of the synapse is a graph showing miniature potentials reaching postsynapse $\mathrm{D}$ at rest and a large amplitude potential during artificial stimulation (the axes are not scaled and the graph is only intended to show the difference). Semblance formation is expected of systems where neurons at certain orders are activated in an oscillating manner.

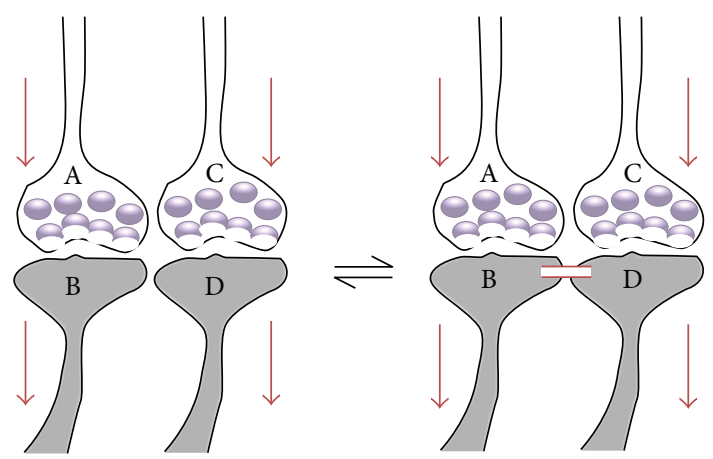

FIGURE 3: Hypothesized formation of a reversible, yet stabilizable, interpostsynaptic functional LINK during associative learning. The simultaneous arrival of activity at the synapses [A-B] and [C-D] induces a reversible functional LINK between their postsynapses $B$ and $\mathrm{D}$. The mechanism of functional LINK formation may include interpostsynaptic membrane hemifusion, ephaptic transmission between the postsynaptic terminals, and still unknown mechanisms.

the neurons, there is a unidirectional flow of activity with a synaptic delay of 1 to 2 milliseconds. In the context of this comparatively huge delay, we anticipate that the systems property of formation of internal sensation is likely independent of the mode of conduction along the neuronal processes. Therefore, replicating the mechanism using electronic circuits by introducing a delay at the nodes between the neuronal units is expected to produce anticipated the results. The systems property of semblance formation is expected to occur if the system is made of neurons that operate with an oscillatory type of activity at certain neuronal orders mimicking the nervous system. With the advances in the field of electronics, integrated circuits built from basic units of operations can be used to test the hypothesis of formation of internal sensations. A previous work has shown how these units can be assembled to obtain systems properties [33].

\section{Interpostsynaptic Functional LINK and LTP}

It is expected that any mechanism for memory storage and retrieval should be able to explain the experimental finding of long-term potentiation (LTP) $[39,40]$ and the observed correlation between LTP and the behavioral motor activities that are surrogate markers for the formation of internal sensation of retrieved memories. Even though classical demonstration of LTP induction is demonstrated in the hippocampal CA3CA1 region, LTP has been demonstrated in various locations in the nervous system. When a stimulating electrode stimulates large number of CA3 neuronal axons (Schaffer collaterals) (Figure 5(a)) whose terminals synapse with dendritic spines of the neurons of the CA1 layer, a recording electrode placed close to the main dendritic stems of the CA1 neurons records electrical changes [41]. The electrical settings of the input electrode are adjusted until the output neuron fires 50\% of the time (labeled as "amplitude of 50\%"). If a brief repetitive stimulation is applied initially at the Schaffer collaterals, then application of a regular stimulus is sufficient to induce a potentiated effect when recorded from the CA1 region 


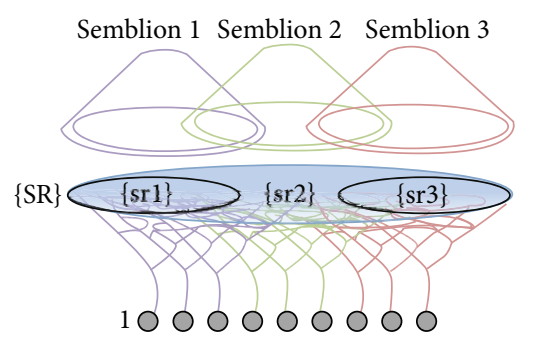

2000000

$3 \bigcirc \bigcirc \bigcirc \bigcirc\{X\}$

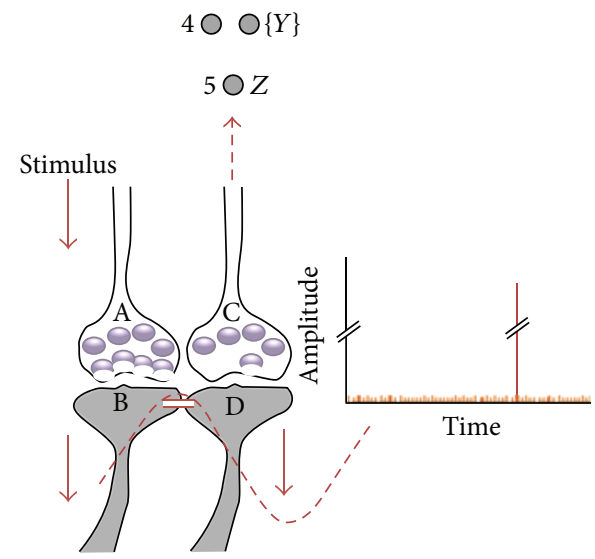

FIGURE 4: Diagrammatic representation of reactivation of an interpostsynaptic functional LINK leading to the formation of semblances at postsynapse D. Instead of directly stimulating postsynapse $\mathrm{D}$ as shown in Figure 2, lateral entry of activity reaching postsynapse D through interpostsynaptic functional LINK can induce semblance. When the cue stimulus used in the associative learning arrives at the presynaptic terminal $\mathrm{A}$ it activates the synapse [AB], reactivates the interpostsynaptic functional LINK, and activates the LINKed postsynapse D. Lateral entry of activity activating the postsynapse $\mathrm{D}$, in the absence of arrival of activity from its presynaptic terminal $\mathrm{C}$, induces semblance of arrival of activity from its presynaptic terminal $\mathrm{C}$ as explained in Figure 2. In systems with junctions where continuous unidirectional quantal release takes place, semblance formation (as explained in Figure 2) at the interLINKed postsynapses is expected as their intrinsic property. On the right side of the synapse is a graph showing potentials reaching the postsynapse $\mathrm{D}$ at rest and a large potential during the arrival of the cue stimulus (they are not scaled and only intended to show the difference). The arrows show the direction of propagation of activity. The waveform indicates the basis for oscillatory neuronal activities where the lateral entry of activity through the interpostsynaptic functional LINK provides the horizontal component and the synaptic transmission provides the vertical component (figure modified from [33]).

and is called LTP. By keeping the experimental preparation viable following the initial brief repetitive stimulation, a regular stimulus applied at the Schaffer collaterals continues to induce LTP for nearly 24 hours. Later, patch clamping the postsynaptic CA1 neuronal soma was used $[42,43]$ to record LTP by stimulating the Schaffer collaterals. A mechanistic explanation is required to explain the rapid induction and slow weaning off of LTP during these procedures. In addition, it should be able to explain how the mechanism can substantiate the observed correlation between the experimental finding of LTP and behavioral motor activities representing the internal sensation of memory. Therefore, it is necessary to explain how the formation of reversible interpostsynaptic functional LINKs can explain LTP.

Continued incremental associative learning within the cage environment leads to the inter-LINKing of many postsynapses in the hippocampi. The group of inter-LINKed postsynapses is called an islet of LINKed postsynapses (Figure 5(a)). During LTP induction, many postsynapses get functionally LINKed due to the simultaneous activation of abutted postsynapses. Some of these LINKs is expected to occur between the islets of already LINKed postsynapses, leading to the generation of mega-islets (Figure 5(b)). Following the induction of LTP, activation of one or more postsynapses by a regular stimulus (not high frequency) can lead to the spread of depolarization between large numbers of inter-LINKed postsynapses within the megaislet. Regular stimuli can now travel through a large number of postsynapses and find more routes to reach the dendritic tree of a single CA1 neuron simultaneously (Figure 5(c)). This results in the maintenance of LTP that can be recorded by both extracellular and patch clamping methods. Membrane hemifusion between the postsynapses is a possible mechanism for the formation of interpostsynaptic functional LINK. Many studies have shown that dendritic spines (postsynapses) swell following LTP and after associative learning [44-47]. As the postsynapses increase in size, their probabilities to abut each other and hemifuse increase. Attenuation of LTP was observed by injection of synaptosomal-associated protein (SNAP) inhibitors that inhibit membrane fusion, into the cell body of CA1 neuron [48]. Since (a) SNAP proteins are present within the postsynapses, (b) postsynaptic membranes are not as freely mobile as the membrane-bound spherical vesicles within the extracellular matrix volume, and (c) postsynaptic membrane curvature is less than that of the membrane-bound spherical vesicles; the hypothesized interpostsynaptic membrane hemifusion (as opposed to membrane fusion) is expected to be blocked by SNAP inhibitors. This provides further support for the proposal of reversible interpostsynaptic membrane hemifusion during LTP.

\section{Electronic Circuit Model for the Interpostsynaptic Functional LINK}

Building and assembling the basic electronic circuit units where the unit of internal sensation is expected to form requires an understanding of the known neuronal connections. The human nervous system has nearly $10^{11}$ neurons. Each neuron has a cell body and is connected with other neurons through incoming terminals (input terminal terminals, also known as postsynaptic terminals or postsynapses or dendritic spines) and outgoing terminals (presynaptic terminals or presynapses) (Figure 6). The number of these terminals varies widely among different neurons ranging from one to tens of thousands [49]. Synapses are the junctions between the output terminals (presynaptic terminals) of 


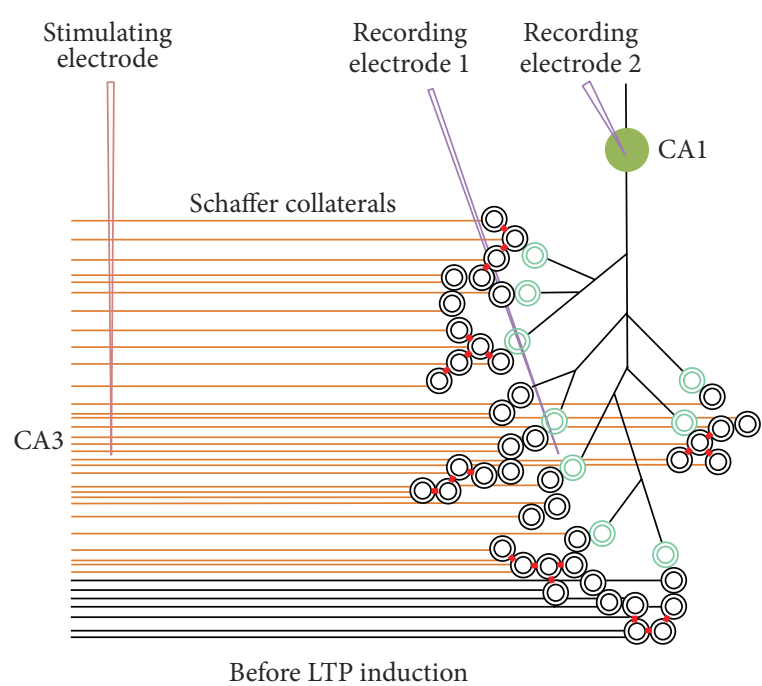

(a)

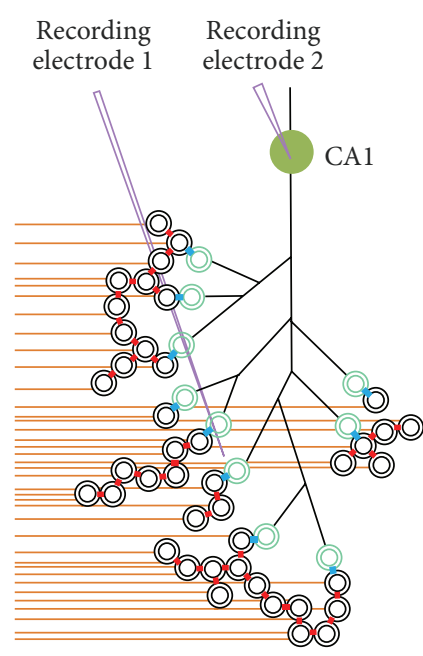

LTP induction

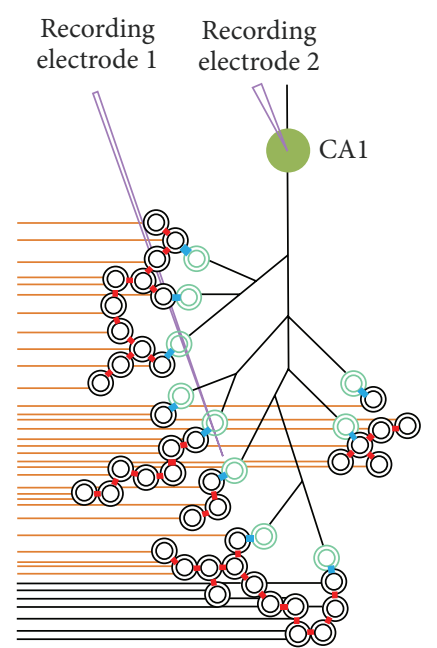

After LTP induction

(c)

FIGURE 5: Illustration explaining long-term potentiation (LTP) based on the formation of interpostsynaptic functional LINKs. (a) Regular stimulation of the Shaffer collaterals (stimulated fibers are shown in orange) before inducing LTP. None of the postsynapses (double rings in green) of the CA1 neuron are inter-LINKed with other postsynapses belonging to other CA1 neurons (not shown). Note the presence of existing interpostsynaptic functional LINKs (in red) forming islets of inter-LINKed postsynapses between postsynapses (double rings in black) that belong to other CA1 neurons that are not shown in the figure. Since there are no functional LINKs that connect to the postsynapses of the shown CA1 neuron, regular stimulation at the Schaffer collaterals will not reach the soma of the shown CA1 neuron. If some of the postsynapses of the CA1 neuron from where we record make few functional LINKs connecting to the postsynapses that are activated by stimulation at the Schaffer collaterals, then the response can be recorded from the given CA1 neuron; however, it will not show any potentiated effect. (b) During LTP induction, high frequency stimulation of the Schaffer collaterals results in formation of a large number of interpostsynaptic functional LINKs between abutted dendritic spines that belong to different CA1 neurons (all stimulated Schaffer collaterals are in orange). Note that all the postsynapses are activated to form reversible interpostsynaptic functional LINKs between them (in red, those interpostsynaptic functional LINKs formed with the postsynapses of the CA1 neuron are shown in blue). Potentials traversing through all the interpostsynaptic functional LINKs and finally reaching the dendritic spines of the shown CA1 neuron result in a potentiated effect when recorded from this CA1 neuron. Swelling of the dendritic spines [44-47] can favor interpostsynaptic membrane hemifusion and blockers of lipid membrane fusion can attenuate LTP [48]. Also note that LTP can be recorded from either extracellular recording (recording electrode 1) or patch clamp recording of the neuronal soma (recording electrode 2). (c) Immediately following LTP induction, a regular stimulus can reach the CA1 neuron through the LTP-induced interpostsynaptic functional LINKs showing a potentiated effect. Since the newly formed interpostsynaptic functional LINKs are reversible, their reversal over time causes gradual decline in the strength of LTP. Postsynapses are shown in double rings to represent membrane bilayer in a cross-sectional view. Short red boxes between the postsynapses indicate interpostsynaptic functional LINKs. Short blue boxes represent the interpostsynaptic functional LINKs to the postsynapses of the CA1 neuron. Presynaptic terminals are not shown. For convenience, stimulating electrode is not shown in (b) and (c).

a neuron and usually one of the input terminals (postsynaptic terminals) of the next order neuron. Nerve conduction is transmitted from one neuron to other neurons through the synapses. In excitatory neurons, synaptic activity produces an excitatory postsynaptic potential (EPSP) at one of the postsynaptic terminals of the neuron and it propagates towards the latter's axonal hillock. For the purpose of this work, only excitatory synapses are used since inhibitory neurons and glial cells can be incorporated into the main framework for fine regulation at various neuronal orders. When a threshold number of EPSPs (nearly forty) arrive (Figure 7 in [50]), they can summate spatially or temporally to induce an action potential, by an "all or none" principle. When the sum of the EPSPs is less than the threshold, it will fall short of inducing an action potential. Even if the sum of EPSPs is much higher than the threshold, it will only induce the same action potential. Once elicited, the action potential propagates towards all the output terminals of the neuron.

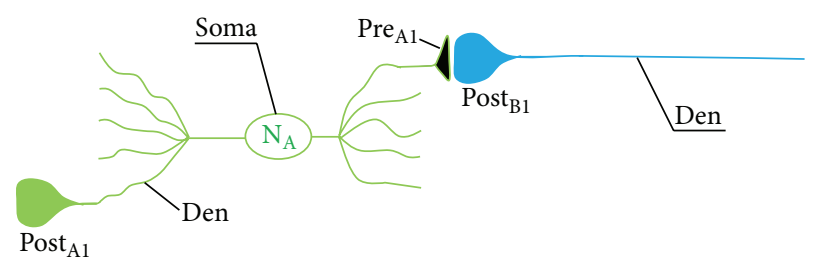

Figure 6: Input and output connections of a neuron. Post ${ }_{\mathrm{A} 1}$ : postsynaptic terminal (postsynapse or dendritic spine) of the first neuron; Den: dendrite; Pre $_{\mathrm{A} 1}$ : presynaptic terminal of the first neuron $\mathrm{N}_{\mathrm{A}}$; Post $\mathrm{B}_{\mathrm{B} 1}$ : postsynaptic terminal of the second neuron $\mathrm{N}_{\mathrm{B}}$ (not shown). The junction between the first and second neurons $\left[\right.$ Pre $_{\mathrm{A} 1}-$ Post $\left._{\mathrm{B} 1}\right]$ forms the synapse. Den: dendrite. Soma: cell body of the neuron $\mathrm{N}_{\mathrm{A}}$.

Interpostsynaptic functional LINK is modeled using an electronic circuit with an aim to replicate the hypothesized changes during associative learning and to demonstrate 
the formation of the internal sensation of memory at the time of memory retrieval. Representative diagrams showing the interpostsynaptic functional LINK and the components of the corresponding electronic circuit are shown in Figures 7(a) and $7(b)$.

A detailed electronic circuit is shown in Figures 8(a) and 8 (b). The input terminals are represented by voltage inputs numbered from $V_{\text {In }} 1, V_{\text {In }} 2, V_{\text {In }} 3, V_{\text {In }} 4$, and $V_{\text {In }} 5$ to the neuron $\mathrm{N}_{\mathrm{A}}$ and $V_{\text {In }} 6, V_{\text {In }} 7, V_{\text {In }} 8, V_{\text {In }} 9$, and $V_{\text {In }} 10$ to the neuron $\mathrm{N}_{\mathrm{C}}$. Neurons have varying number of terminals ranging from one, at some of the early orders of neurons in visual pathway, to nearly 2.4 to $5.2 \times 10^{4}$ input terminals (postsynaptic terminals receiving EPSPs) to a neuron in higher neuronal orders [49]. These neuronal inputs are represented by five input terminals to each neuron $\mathrm{N}_{\mathrm{A}}$ and $\mathrm{N}_{\mathrm{C}}$. When an excitatory synapse is activated, it induces EPSP at the postsynaptic terminal. A regulated direct current power supply is used to provide constant voltage to the five input terminals $V_{\text {In }} 1, V_{\text {In }} 2, V_{\text {In }} 3$, $V_{\text {In }} 4$, and $V_{\text {In }} 5$ of the neuron $\mathrm{N}_{\mathrm{A}}$ and $V_{\text {In }} 6, V_{\text {In }} 7, V_{\text {In }} 8, V_{\text {In }} 9$, and $V_{\text {In }} 10$ of the neuron $\mathrm{N}_{\mathrm{C}} \cdot R_{1}, R_{2}$ and $R_{3}, R_{4}$ are representative resistor sets shown at each one of the input terminals of the neurons $\mathrm{N}_{\mathrm{A}}$ and $\mathrm{N}_{\mathrm{C}}$ whose values can be adjusted to determine the voltage at each input. The origin of action potential generation is modeled using the voltage comparator OpAmps marked as $\mathrm{N}_{\mathrm{A}}$ and $\mathrm{N}_{\mathrm{C}}$. Resistors $R_{5}$ and $R_{6}$ form a voltage divider that determine $V$ - value to the comparator. The resistor values are set up such that when the number of inputs to the $V+$ of the comparator is less than two, no output (action potential) is generated. If the number of inputs is two or more than two, an output is generated. Even if $\mathrm{N}_{\mathrm{A}}$ or $\mathrm{N}_{\mathrm{C}}$ receives all five inputs, they elicit only one output (action potential). This makes the output function of the voltage comparator OpAmp similar to the functioning of "all or none" phenomenon of action potential generation of a neuron:

$$
\text { Action potential }(n)= \begin{cases}1, & \text { if } x_{i} n \geq 2, \\ 0, & \text { if } x_{i} n<2\end{cases}
$$

This is represented by the $V_{\text {Out }}$ of the comparators as follows:

$$
\begin{aligned}
& \text { High } \longleftarrow \sum V_{\text {In }}>V- \\
& \text { Low } \longleftarrow \sum V_{\text {In }}<V-
\end{aligned}
$$

Before an associative learning event, independent activation of individual neurons $\mathrm{N}_{\mathrm{A}}$ and $\mathrm{N}_{\mathrm{C}}$ results in the generation of independent activity at their corresponding postsynaptic dendritic terminals $V_{\text {Out }} 1$ and $V_{\text {Out }} 2$ that are shown as the outputs of the circuit unit. During associative learning, when neurons $\mathrm{N}_{\mathrm{A}}$ and $\mathrm{N}_{\mathrm{C}}$ are activated together, it results in the formation of an interpostsynaptic functional LINK. After associative learning, the arrival of activity through one of the neuronal pathways results in the reactivation of the interpostsynaptic functional LINK. This results in the flow of activity through both the postsynaptic dendrites $V_{\text {Out }} 1$ and $V_{\text {Out }} 2$.

The synapse $\left[\mathrm{Pre}_{\mathrm{A1}}-\right.$ Post $_{\mathrm{B1}}$ ] is represented by the diodes $D_{1}, D_{2}$, and $D_{3}$ and the changes taking place between them.
The second synapse [Pre ${ }_{\mathrm{C} 1}-$ Post $_{\mathrm{D} 1}$ ] is represented by the set of diodes $D_{4}, D_{5}$, and $D_{6}$ and the changes taking place between them. Changes taking place at these diodes representing the synapse include unidirectional synaptic transmission, a synaptic delay of 1 to 2 milliseconds, and the potential changes resulting from quantal release from the presynaptic terminal to the synaptic cleft. A synaptic delay of 1 to 2 milliseconds is achieved in the synapses [Pre ${ }_{\mathrm{A} 1}-$ Post $\left._{\mathrm{B} 1}\right]$ and $\left[\mathrm{Pre}_{\mathrm{C} 1}-\right.$ Post $_{\mathrm{D} 1}$ ] by using the resistor capacitor sets $R_{7}, C_{1}$ and $R_{12}, C_{2}$, respectively. The changes in the potential difference between the plates of capacitors $C_{1}$ and $C_{2}$ through the resistors $R_{7}$ and $R_{12}$, respectively, are given by the formula $V=V_{r}+\left(V_{o}-\right.$ $\left.V_{r}\right) e^{-(t / R C)}$, where $V=$ voltage on the capacitor at time $t ; V_{r}=$ voltage from $D_{1}$ or $D_{4} ; V_{o}=$ initial voltage on the capacitor; $t=$ charging or discharging time; $R=$ resistance; $C=$ capacitance. Synaptic delay of the circuit depends on the time needed to charge the capacitor located at the presynaptic terminal. The cathodes of the diodes $D_{3}$ and $D_{6}$ represent the postsynaptic terminal. Neurons are represented by the comparators $\mathrm{N}_{\mathrm{A}}$ and $\mathrm{N}_{\mathrm{C}}$, respectively. When $\mathrm{N}_{\mathrm{A}}$ and $\mathrm{N}_{\mathrm{C}}$ are activated, all their output terminals (presynaptic or axonal terminals) receive voltage. Pre $_{\mathrm{A} 1}$ and $\mathrm{Pre}_{\mathrm{C} 1}$ are the two presynaptic terminals, respectively, of the neurons $\mathrm{N}_{\mathrm{A}}$ and $\mathrm{N}_{\mathrm{C}}$. Pre ${ }_{\mathrm{A} 1}$ is represented by the combination of diodes $D_{1}, D_{2}$ and the anode of the diode $D_{3}$. Pre $\mathrm{C}_{\mathrm{C} 1}$ is represented by the combination of diodes $D_{4}, D_{5}$ and the anode of the diode $D_{6}$. Post ${ }_{\mathrm{B} 1}$ and Post $\mathrm{D}_{\mathrm{D} 1}$ are the two postsynaptic terminals and are represented by the cathodes of the diodes $D_{3}$ and $D_{6}$. When activity reaches a synapse, it elicits an EPSP at the postsynaptic terminal (input terminal) of the next neuron.

The synapses at the output terminals (axonal terminals) Pre $_{\mathrm{A} 1}$ and Pre $_{\mathrm{C} 1}$ of neurons $\mathrm{N}_{\mathrm{A}}$ and $\mathrm{N}_{\mathrm{C}}$, respectively, are abutted to each other at various locations in the nervous system where inputs converge. When these synapses are activated simultaneously during associative learning, a functional LINK is formed between their respective postsynaptic terminals Post ${ }_{\mathrm{B} 1}$ and Post ${ }_{\mathrm{D} 1}$. In the electronic circuit, this is represented by the formation of a reversible LINK between the cathodes of the diodes $D_{3}$ and $D_{6}$. The interpostsynaptic functional LINK [ Post $_{\mathrm{B} 1}$-Post ${ }_{\mathrm{D} 1}$ ] is primarily represented by the operation of the latch marked Latch1. When both neurons $\mathrm{N}_{\mathrm{A}}$ and $\mathrm{N}_{\mathrm{C}}$ are activated together, the output terminals are functionally LINKed by the arrival of sufficient activity at the AND gate AND1 and the latch marked Latch1. Once triggered, Latchl continuously provides output. This is equivalent to the formation and maintenance of an interpostsynaptic functional LINK as long as input arrives at the Post ${ }_{\mathrm{B} 1}$. Once arrival of the input at Post $_{\mathrm{B} 1}$ stops, the interpostsynaptic functional LINK remains in a reactivatible state following an associative learning event. The strength of the interpostsynaptic functional LINK is increased by mechanisms that can stabilize it. As the stability increases, the duration of memory storage increases. The reset function of the Latch1 is controlled by the timer marked Timerl that determines the duration of the function of the latch. This guides the adjustment of the duration of operation of the system. Once triggered, the constant output from the Latchl continuously reaches one of the inputs to the second AND gate AND2. At a later time, the arrival of sufficient stimuli capable of activating 


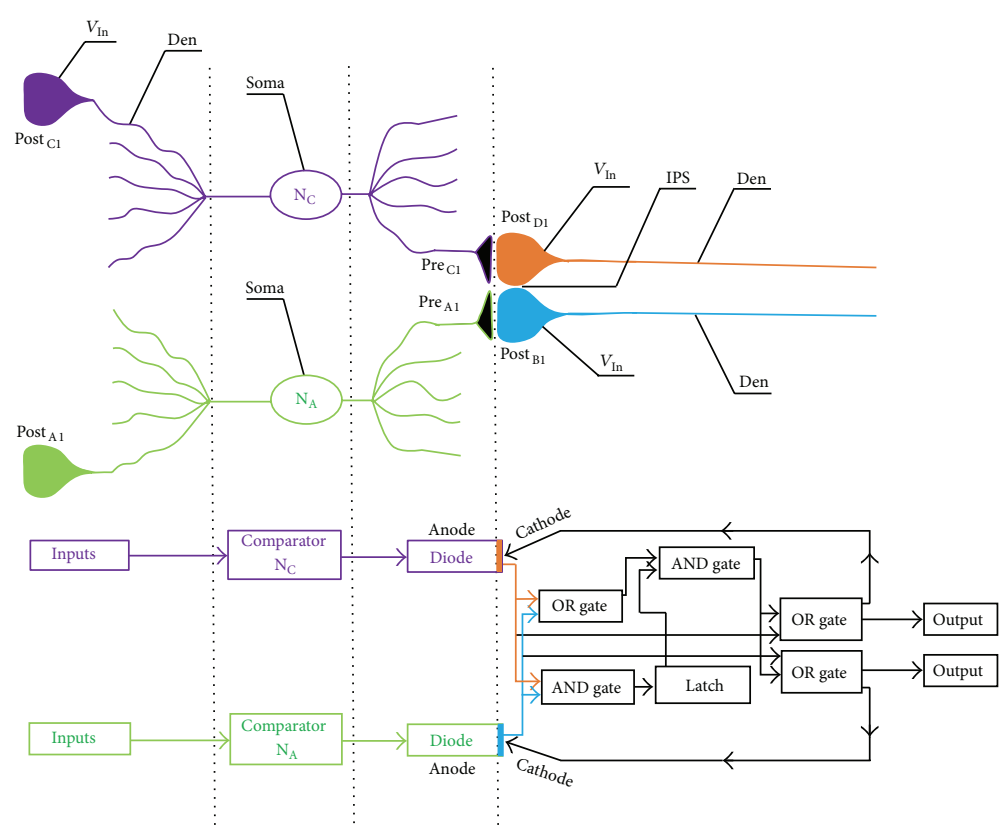

(a)

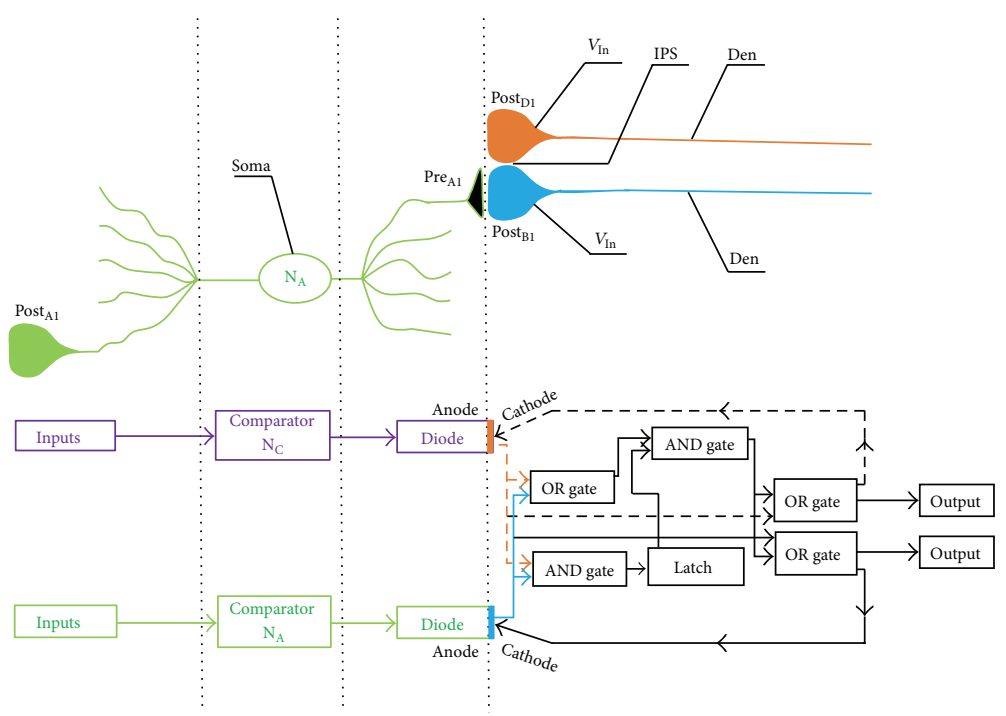

(b)

FIGURE 7: Comparative diagrams showing interpostsynaptic functional LINK and its corresponding electronic circuit at the time of both formation and reactivation of the interpostsynaptic functional LINK. (a) Schematic diagram showing a comparison between a neuronal circuit (above) and the corresponding electronic circuit (below) showing the formation of a functional LINK between the postsynaptic terminals at the time of associative learning. Simultaneous activation of abutted postsynapses results in interpostsynaptic functional LINKs which is modelled using the latch that can be controlled through a timer. The detailed electronic circuit is shown in Figure $8 . \mathrm{N}_{\mathrm{A}}$ and $\mathrm{N}_{\mathrm{C}}$ are presynaptic neurons. Cell bodies of postsynaptic neurons are not shown. Synapses are marked as $\left[\mathrm{Pre}_{\mathrm{A1}}-\mathrm{Post}_{\mathrm{B1}}\right]$ and $\left[\mathrm{Pre}_{\mathrm{Cl}}-\mathrm{Post}_{\mathrm{D} 1}\right]$. Outputs occur through both the output terminals (dendrites from both Post $_{\mathrm{B} 1}$ and Post ${ }_{\mathrm{D} 1}$ ). (b) A schematic diagram showing a comparison between a neuronal circuit and the corresponding electronic circuit showing the reactivation of the interpostsynaptic functional LINK by the cue stimulus arriving at neuron $\mathrm{N}_{\mathrm{A}}$ at the time of memory retrieval. Two major changes are expected to occur at this time as a systems property. These include $(A)$ semblions getting induced at the cathode of the diode located immediately after the comparator $N_{C}$ and (B) activity then spreading to the postsynapse Post $\mathrm{D}_{1}$. Post $\mathrm{A}_{\mathrm{A}}$ : postsynapse of the neuron $\mathrm{N}_{\mathrm{A}}$. Pre $\mathrm{P}_{\mathrm{A} 1}$ : presynapse (axonal terminal) of the neuron $\mathrm{N}_{\mathrm{A}}$. Post $\mathrm{Cl}_{\mathrm{C}}$ : postsynapse of the neuron $\mathrm{N}_{\mathrm{C}}$. Post $\mathrm{B}_{\mathrm{B}}$ : postsynapse of the neuron $\mathrm{N}_{\mathrm{B}}$ (not shown). Post $\mathrm{D}_{1}$ : postsynapse of the neuron $\mathrm{N}_{\mathrm{D}}$ (not shown). IPS: interpostsynaptic functional LINK. Den: dendrite. During associative learning simultaneous activation of abutted postsynapses. 


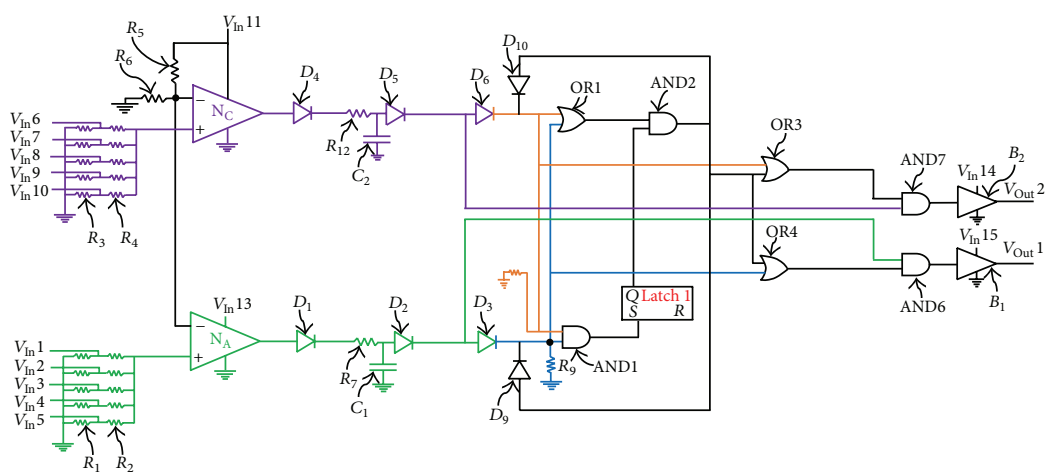

(a)

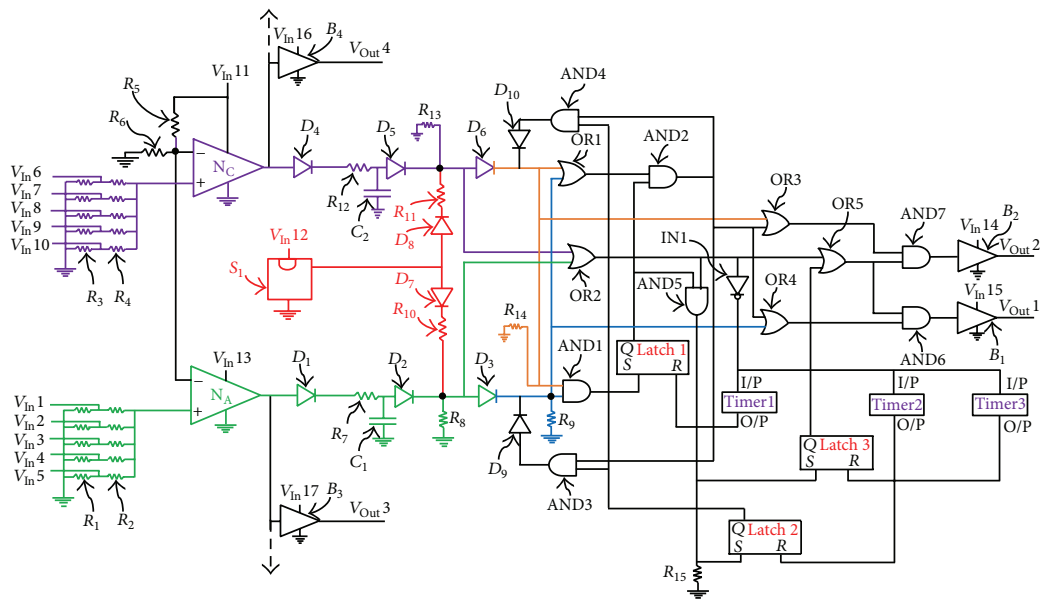

(b)

FIGURE 8: Electronic circuit model for the interpostsynaptic functional LINK, which is shown in Figures 3 and 7. (a) Preliminary electronic circuit in the process of building the final circuit model of the interpostsynaptic functional LINK shown in (b). Simultaneous activation of two different neurons $\mathrm{N}_{\mathrm{A}}$ and $\mathrm{N}_{\mathrm{C}}$ (in green and violet, resp.) results in the formation of a functional LINK between the postsynaptic terminals at the synapses located at each one of their output terminals (presynaptic terminals). When neuron $\mathrm{N}_{\mathrm{A}}$ is activated alone before associative learning, the output from the system occurs through $V_{\text {Out }} 1$, since both inputs of the logic gate AND6 receive current from the neuron $\mathrm{N}_{\mathrm{A}}$. Similarly, as long as neuron $\mathrm{N}_{\mathrm{C}}$ is activated alone, output from the system occurs through $V_{\text {Out }} 2$, since both inputs of the logic gate AND7 receive current from neuron $\mathrm{N}_{\mathrm{B}}$. The interpostsynaptic functional LINK is represented by Latchl. When neurons $\mathrm{N}_{\mathrm{A}}$ and $\mathrm{N}_{\mathrm{C}}$ are activated simultaneously, Latch1 gets latched. Since the duration of the latch is not controlled, Latch1 remains latched and the logic gate AND2 will keep on receiving one of its inputs. Since many of the newly formed interpostsynaptic functional LINKs have half-lives, fine regulation of the duration of the interpostsynaptic functional LINKs is required to represent them and is shown in (b). After associative learning that involves simultaneous activation of neurons $\mathrm{N}_{\mathrm{A}}$ and $\mathrm{N}_{\mathrm{C}}$ in their pathways, activation of either one of the neurons $\mathrm{N}_{\mathrm{A}}$ or $\mathrm{N}_{\mathrm{C}}$ by either one of the stimuli that took part in associative learning is sufficient to provide the second input to AND2 through the logic gate OR1. When AND2 is activated, it activates the postsynaptic terminals of both synapses that are LINKed through the interpostsynaptic functional LINK. The activation of a postsynapse by activity entering from the lateral side in the absence of arrival of activity from its corresponding presynaptic terminal results in semblances, cellular hallucinations about possible features of the item whose memory is retrieved. Semblance formation is expected to occur in systems when (a) unidirectional activation occurs at the synapses, (b) continuous changes in potential of the postsynaptic terminal occur from quantal release of neurotransmitter from its presynaptic terminal, and (c) lateral entry of activity through the interpostsynaptic functional LINK contributes to the horizontal component of the oscillating neuronal activity; $V_{\text {In }}=V_{\text {Out }}$ (b) This circuit diagram is a modification of the circuit shown in (a). It has a voltage source S1 (in red) for providing pulses of miniature voltage changes due to quantal release from the presynaptic terminals that are represented in the circuit by the anodes of the diodes $D_{3}$ and $D_{6}$. The additional features include controls for adjusting the duration of interpostsynaptic functional LINKs, the duration of outputs through the postsynaptic terminal dendrite, and the duration of reactivation of the functionally LINKed postsynaptic terminals, providing a flexible circuit to simulate the functioning of the nervous system. Once an interpostsynaptic functional LINK is formed, it remains in a dormant state for a certain period of time depending on its life-span. There are three latches, Latch1, Latch2, and Latch3. Latch1 represents the dormant interpostsynaptic functional LINK. If sensory inputs do not arrive through the neurons $\mathrm{N}_{\mathrm{A}}$ or $\mathrm{N}_{\mathrm{C}}$, then the OR gate OR2 is closed. This activates the inverter IN1, which in turn activates three downstream timers Timer1, Timer2, and Timer3 that in turn control three downstream events. (1) The triggering of Timer1 controls the duration of functioning of Latch1, which represents the interpostsynaptic functional LINK. (2) The triggering of Timer2 controls the duration of functioning of Latch2 that determines the duration of activation of the postsynaptic terminals that are represented by the cathodes of diodes $D_{3}$ and $D_{6}$ (Diodes $D_{3}$ and $D_{6}$ represent synapses). (3) The triggering of Timer 3 controls the duration of functioning of Latch 3 that determines the duration of activity at postsynaptic dendrites $V_{\text {Out }} 1$ and $V_{\text {Out }} 2$. Additional presynaptic terminals $V_{\text {Out }} 3$ and $V_{\text {Out }} 4$, respectively, of neurons $\mathrm{N}_{\mathrm{A}}$ and $\mathrm{N}_{\mathrm{C}}$ are also shown; $V_{\text {In }}=V_{\text {Out }}$. 
either one of the neurons $\mathrm{N}_{\mathrm{A}}$ or $\mathrm{N}_{\mathrm{C}}$ through the OR gate $\mathrm{OR} 1$ provides the second input to activate AND2. Activation of the AND2 represents the reactivation of the interpostsynaptic functional LINK.

When neuron $\mathrm{N}_{\mathrm{A}}$ is activated following associative learning, it reactivates the interpostsynaptic functional LINK [Post ${ }_{\mathrm{B} 1}$-Post $\left.{ }_{\mathrm{D} 1}\right]$ that was formed during associative learning and the voltage reaches the cathode of the diode $D_{6}$ that represents the functionally LINKed postsynaptic terminal of neuron $\mathrm{N}_{\mathrm{C}}$. This arrival of activity at the cathode of diode $D_{6}$ in the absence of the arrival of activity from its anode is expected to produce a cellular hallucination that it is being activated by the anode of the diode $D_{6}$ as a systems property. Activating a postsynaptic terminal without arrival of activity from its corresponding presynaptic terminal is expected to form basic units of internal sensations as a systems property when a large number of units are arranged similar to arrangements found in the nervous systems of different animal species.

Normal synaptic transmission takes place through the release of neurotransmitter from a large number of synaptic vesicles from the presynaptic terminal only at the time of arrival of neuronal activity. In contrast, quantal release of neurotransmitters from single vesicles from the presynaptic terminal continuously elicits miniature potentials at the postsynaptic terminal even at rest. These contribute towards the recordable miniature EPSPs (mEPSPs) at the soma. The voltage generated by quantal release at rest generating mEPSP is substantially lower than the voltage induced by EPSP generated by the arrival of an action potential at the presynaptic terminal:

miniature potentials at the postsynaptic terminal induced by the release of contents of a single synaptic vesicle «EPSP at the postsynaptic terminal induced by the arrival of action potential at the presynaptic terminal

The voltage source for mEPSP is represented as follows. Timer IC marked as S1 provides pulsed intermittent miniature voltages through the sets of diode and resistor $D_{8}$ and $R_{11}$ and $D_{7}$ and $R_{10}$. The miniature voltages at the anode of the diodes $D_{3}$ and $D_{6}$ continuously cross to the cathode side that represents the postsynaptic terminals of the synapses. Due to the miniature nature of these voltages, they do not contribute towards the activation of the neurons in the next neuronal order. The continuous arrival of miniature voltage at the cathodes (that represents the postsynaptic terminal) of the diodes (that represent the synapse) $D_{3}$ and $D_{6}$ even at rest is a unique feature of the system. This context is essential for the formation of cellular hallucination of arrival of activity from corresponding presynaptic terminal when activity arrives at the postsynaptic terminal through the interpostsynaptic functional LINK.

Additional presynaptic terminals from the neurons $\mathrm{N}_{\mathrm{A}}$ and $\mathrm{N}_{\mathrm{C}}$ are also shown and are represented by $V_{\text {Out }} 3$ and $V_{\text {Out }} 4$ from the buffers $B_{3}$ and $B_{4}$, respectively. Formation of interpostsynaptic functional LINKs at the postsynaptic terminals of the synapses that are formed at the additional output terminals enables this electronic circuit unit to be expanded and interconnected with large number of similar units. Following associative learning the interpostsynaptic functional LINK remains active, which is represented by the state of the latch marked as Latchl to remain as latched. At this time arrival of activity through either one of the neurons $\mathrm{N}_{\mathrm{A}}$ or $\mathrm{N}_{\mathrm{B}}$ activates the latches marked Latch2 and Latch3. This is facilitated through the AND gate AND5. The arrival of activity through either one of the inputs activates Latchl and provides the second input to the AND gates AND3 and AND4. This part of the circuit provides control of the duration of activation of the functionally LINKed postsynaptic terminals. Arrival of activity through either one of the inputs activates the latch marked Latch 2 and provides the second input to the OR gate OR5. This part of the circuit provides output from the circuit unit. When the AND gate AND2 is activated, it simultaneously provides output from the unit through both the outputs $V_{\text {Out }} 1$ and $V_{\text {Out }} 2$. Note that by using the buffers $B_{1}$ and $B_{2}$, steady voltage is made to arrive at the postsynaptic dendrites $V_{\text {Out }} 1$ and $V_{\text {Out }} 2$. The outputs at $V_{\text {Out }} 1$ and $V_{\text {Out }} 2$ are essential for downstream neuronal activation.

When neurons $\mathrm{N}_{\mathrm{A}}$ and $\mathrm{N}_{\mathrm{C}}$ are activated simultaneously, an interpostsynaptic functional LINK [Post ${ }_{\mathrm{B} 1}-$ Post $_{\mathrm{D} 1}$ ] is formed. When this functional LINK is not reactivated, it remains in a dormant state for a certain period of time depending on its life-span. The latch marked Latch1 represents the dormant interpostsynaptic functional LINK. Following associative learning, if sensory inputs do not arrive through the neurons $\mathrm{N}_{\mathrm{A}}$ or $\mathrm{N}_{\mathrm{C}}$, then the OR gate OR2 gets closed. This activates the inverter IN1, which in turn activates three downstream timers Timer1, Timer2, and Timer3. This event controls three downstream events. (1) Triggering the Timerl controls the duration of functioning of Latch1, which represents the interpostsynaptic functional LINK. (2) Triggering Timer 2 controls the duration of functioning of Latch2 and determines the duration of activation of the postsynaptic terminals that are represented by the cathodes of the diodes $D_{3}$ and $D_{6}$. (3) Triggering of Timer3 controls the duration of functioning of Latch3 that determines the duration of activity at the postsynaptic dendrites $V_{\text {Out }} 1$ and $V_{\text {Out }} 2$. Latches marked Latch2 and Latch3 are made to function continuously through the arrival of activity along one of the inputs only when the Latchl that represents the interpostsynaptic functional LINK remains activated. If the interpostsynaptic functional LINK represented by Latch1 remains active, then the arrival of activity at any one of the inputs enables the activation of the cathode of the diode that represents the functionally LINKed postsynaptic terminal. Thus, the duration of the interpostsynaptic functional LINKs, the duration of reactivation of the functionally LINKed postsynaptic terminals, and the duration of activation of dendrites of the functionally LINKed postsynaptic terminals are functions initiated by the stoppage of arrival of activity to both the diodes $D_{3}$ and $D_{6}$ that represent synapses of the functionally LINKed postsynaptic terminals.

From the AND gate AND2, activity reaches the postsynaptic terminals of both synapses. These take place through the AND gate AND3 and diode $D_{9}$ towards the postsynaptic terminal of the synapse $\left[\mathrm{Pre}_{\mathrm{A} 1}-\mathrm{Post}_{\mathrm{B} 1}\right]$ represented 
by the cathode of the diode $D_{3}$ and the AND gate AND4 and diode $D_{10}$ towards the postsynaptic terminal of the synapse $\left[\mathrm{Pre}_{\mathrm{Cl}}-\right.$ Post $\left._{\mathrm{D} 1}\right]$ represented by the cathode of the diode $D_{6}$. During memory retrieval, the activation of either one of the input neurons $\mathrm{N}_{\mathrm{A}}$ or $\mathrm{N}_{\mathrm{C}}$ by the cue stimulus leads to the reactivation of the interpostsynaptic functional LINK [ Post $_{\mathrm{B} 1}$-Post $_{\mathrm{D} 1}$ ] that results in the arrival of activity at the cathode (postsynaptic terminal) side of the functionally LINKed postsynaptic terminals $D_{3}$ or $D_{6}$.

This circuit allows maintaining the interpostsynaptic functional LINK until its life-span is over, even in the absence of arrival of continuous activity for continuous activation of either one of the LINKed postsynaptic terminals. Similarly, even in the absence of neuronal output from the unit, this circuit maintains interpostsynaptic functional LINKs until its life-span is over. The life-span of the interpostsynaptic functional LINK is achieved in the circuit through the set time of Timerl. The timers marked Timer 2 and Timer 3 are set for shorter periods of time compared to Timerl. After the life-span of the interpostsynaptic functional LINK is over, the arrival of activity through either of the synapses $D_{3}$ and $D_{6}$ provides outputs only through their corresponding outputs $V_{\text {Out }} 1$ and $V_{\text {Out }} 2$, respectively, as occurred before associative learning. The arrangements of the OR gates OR3, OR4, and OR5 and the AND gates AND6 and AND7 enable the circuit to accomplish these functions.

The functional LINK between the postsynaptic terminals is built using electronic circuits in order to provide all its functional attributes. This consists of (a) inputs reaching the neurons triggering action potential when summated EPSPs surpass a threshold value, (b) action potentials traversing to the output terminals (presynaptic terminals) activating the synapses at the their terminals, and (c) activity reaching the postsynaptic terminals. When the simultaneous activation of closely located postsynaptic terminals of different neurons takes place, it induces a functional LINK between those postsynaptic terminals. The formed interpostsynaptic functional LINK is reactivated by activity arriving at either one of the postsynaptic terminals from their respective inputs. Each interpostsynaptic functional LINK has a specific lifespan. This is represented by a time-period during which an interpostsynaptic functional LINK becomes reactivatible. This reactivation of the interpostsynaptic functional LINK results in the spread of activity towards the postsynaptic terminal of the second synapse that already was functionally LINKed during a previous associative learning event.

4.1. Functional States of the Interpostsynaptic Functional LINK. The functioning of the electronic circuit can be explained as occurring in four states (Figure 9). At the resting state, the activation of neurons $\mathrm{N}_{\mathrm{A}}$ or $\mathrm{N}_{\mathrm{C}}$ results in independent outputs from their corresponding output terminals $V_{\text {Out }} 1$ and $V_{\text {Out }} 2$. The second state is the formation of the functional LINK between the postsynaptic terminals during associative learning, which is represented by the activation of the latch marked as Latchl. The third state occurs when activity stops arriving at both the inputs. This leads to the activation of three timers, which in turn controls
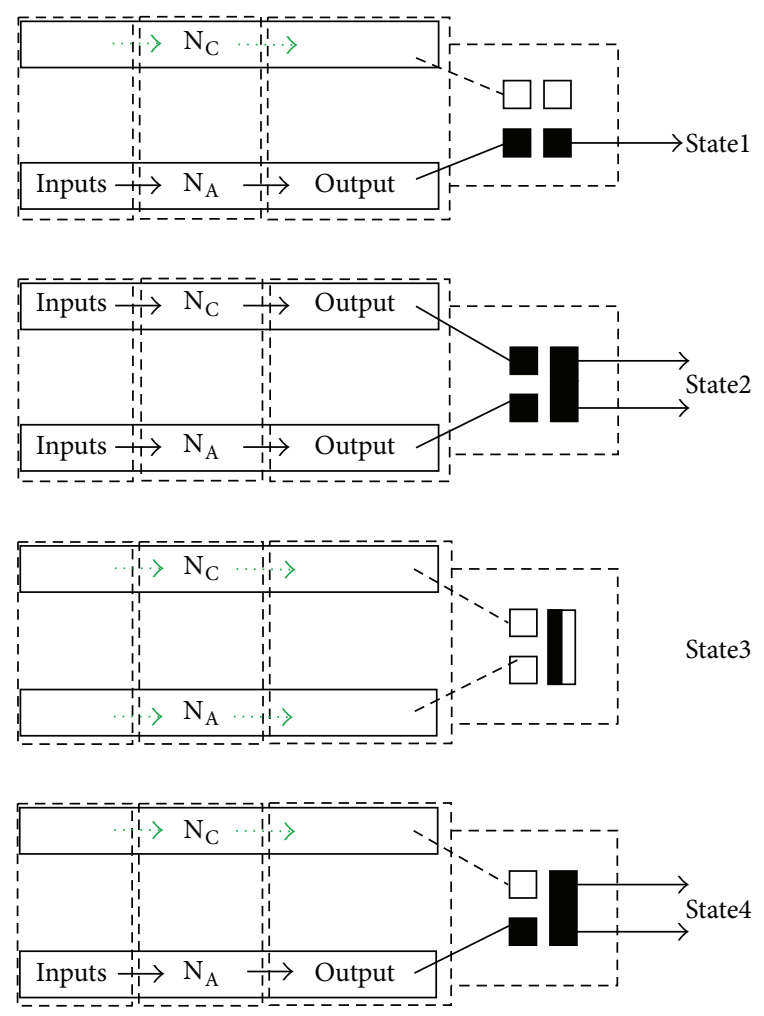

FIGURE 9: Representative diagram showing the functioning of the electronic circuit explained as occurring in four states. STATE1 explains native synaptic function before associative learning is undergone. It demonstrates that even if the postsynaptic terminals of two synapses are abutted to each other, the arrival of activity at one of the postsynaptic terminals alone does not result in any change between the postsynaptic terminals. STATE2 demonstrates that the simultaneous activation of the postsynaptic terminals of two synapses results in the formation of a functional LINK between them. STATE3 demonstrates that once a functional LINK is formed between the postsynaptic terminals by associative learning, it remains dormant until the arrival of activity at one of the postsynaptic terminals occurs. STATE4 demonstrates the reactivation of the previously formed functional LINK between the postsynaptic terminals by the arrival of neuronal activity at one of the postsynaptic terminals $\mathrm{N}_{\mathrm{A}}$ resulting in activation of the second postsynapse. This results in (a) the induction of the semblance of activity arriving from $\mathrm{N}_{\mathrm{C}}$ and (b) the spread of activity in the downstream direction (towards the higher neuronal order) from both the LINKed postsynaptic terminals. The four small boxes with solid sides within the large dotted box on the far right side represent the pre- (left) and postsynapses (right).

the life-span of the interpostsynaptic functional LINK, duration of activation of the LINKed postsynaptic terminals, and activity along the LINKed postsynaptic dendrites towards the cell bodies of their neurons. The fourth state is the reactivation of the interpostsynaptic functional LINK when activity arrives at either one of the postsynaptic terminals that participated in the formation of the interpostsynaptic functional LINK, if the functional LINK remains reactivatible (before its life-span is over). This reactivation of the interpostsynaptic functional LINK activates the LINKed postsynaptic 
terminal resulting in semblance formation and also in the spread of activity along the dendrite of the LINKed postsynaptic terminal. The duration of the reactivatible state of the functionally LINKed postsynaptic terminal is dependent on the duration of existence of the interpostsynaptic functional LINK. The life-span of the interpostsynaptic functional LINK, its existence in a reactivatible state, and the duration of activation of the functionally LINKed postsynaptic terminal are determined by the preset values of the timers Timerl, Timer2, and Timer3, respectively.

4.2. Assembling the Units to Form an Artificial Nervous System. The functional units must be arranged in a similar fashion as found in different nervous systems. A detailed method by which these units can be arranged was explained previously [33]. Apart from the general principles detailed in the above work, it is necessary to provide specific arrangements of the neuronal connections. For example, at the sensory cortices where the sensory inputs arrive, it may be necessary to arrange the inputs in minicolumns for perception. Even though the cortical columns have a network of regulated operation [51], one can begin assembling the units without complex regulatory paths. Initial experiments need only to focus on the general layout of the circuit units. It is expected that the outputs from the primary sensory cortices will converge at locations of convergence of sensory inputs, for example, the hippocampus. At the hippocampus and cortex where large number of interpostsynaptic functional LINKs are expected to be present, the horizontal component provided by the interpostsynaptic functional LINKs can contribute towards the oscillating neuronal activity in a group of neurons. When assembled in large numbers mimicking the nervous system, it is anticipated to have a region similar to the hippocampus when inputs from various sensory systems converge. The system should continuously receive sensory stimuli similar to the background stimuli that are obtained from breathing movements, blood flow, and cutaneous stimulation. The present circuit manually adjusts the timers that control the latches to demonstrate the period during which interpostsynaptic functional LINKs remain reactivatible and also its life-span. In robotic systems, these timers can be automated to operate proportional to the number and strength of the reactivations of the interpostsynaptic functional LINKs.

Convergence of sensory inputs within the temporal lobe can result in formation of large number of interpostsynaptic functional LINKs during associative learning. Depending on the number of inputs converging to an area, it should be possible to induce changes similar to LTP as described in Figure 5 and occlusion experiments carried out in animals [52]. Propagation of activities to further higher neuronal orders can result in sparse convergence that can provide specificity in formation of specific memories. There are a number of areas in the temporal lobe neocortex where multisensory convergence occurs as indicated by the presence of neurons in this area responding to different sensory modalities [53]. This has led to demonstration of different phenomenon such as what is seen affects what is heard [54] and perception of auditory syllables that are influenced by what is seen [55].
4.3. Obtaining Readouts of Internal Sensations. An objective third-person assessment of memories can be best carried out if readouts of the internal sensations can be obtained. This can then be matched with the behavioral motor outputs that we presently use as the surrogate marker for internal sensations. The first-person property of internal sensations expected to form within the machines can be best approached by the following steps [33]. The core principle should be based on deriving the relationship between how a system perceives an object and how that system later retrieves the internal sensations of memories of the object when exposed to a cue stimulus. It involves discovering the computational algorithm using all the semblions at the location of reactivation of interpostsynaptic functional LINKs to match the sensory stimuli that arrived from the item before learning, whose memories are currently being retrieved. When building robotic systems, the first step is to identify the locations of interpostsynaptic functional LINKs that are reactivated in the presence of the cue stimulus. The second step is to find all the possible semblions that can be formed at the functionally LINKed postsynapses that are activated during the reactivation of the interpostsynaptic functional LINKs. The third step is to find an algorithm to compute the correct set of semblions to obtain the net semblance that matches the item whose memories are retrieved.

4.4. Motor Outputs from Robotic Systems. Along with the steps mentioned in the above session, the net semblance is expected to match with the activation of motor neurons that result in behavioral motor activity. In order to identify this, it is required to examine which motor neurons are receiving baseline net EPSP (secondary to oscillating neuronal activity), less than the required threshold EPSP for eliciting action potential. Neurons that are kept inhibited by activities of inhibitory interneurons are also examined. The details of the mechanism that connects the formation of internal sensation and behavioral motor outputs were described previously [20]. A combination of computations for matching semblances along with motor activity can be programmed in robotic systems. This requires adjusting the circuit parameters by methods of trial and error. As it takes many years, following birth, for a human nervous system to mature and to produce expected behavioral motor activity, a given robotic system is expected to require large number of initial associative learning steps before it can provide meaningful outputs. Once the optimal maturation of a given system is achieved, the emerging rules of connectivity can guide building more complex systems faster. In this regard, initial tests need to be carried out to replicate simple nervous systems capable of providing robust motor responses.

\section{Discussion}

Various neural circuit models have been proposed in the past. Completely recurrent neural network model with adaptive synapses [56], item-and-order working memory model that explains how successive items presented through time are stored in working memory as a temporal pattern of activity 
across working memory cells [57], and winner-take-all models with both theoretical [58] and laboratory observations [59] have been examined. However, these models have not explained the nature of the internal sensations being created within them. The present circuit provides provision for the formation of inner sensations expected to form at specific oscillatory firing frequencies of neurons at certain neuronal orders. By varying the frequencies, the nature of the internal sensations can be changed and eventually are expected to stop during sleep. As the proposed model is a dynamic system in principle, stability is a critical issue. The nonlinearity in the model implies that stability of all parts of the circuit may not result in the stability of the overall system. Moreover, continuous formation of new neurons at certain orders of neurons of the circuitry can bring additional instability. Three factors are expected to prevent instability. First, since the firing of neurons takes place by "all or none" phenomena, a large number of inputs that arrive in surplus of the threshold inputs do not have an effect on neuronal firing. This is represented by the operation of the comparator in the electronic circuit. Secondly, at locations where the inputs converge, the lateral spread of activity through the interpostsynaptic functional LINKs is expected to contribute to the horizontal vector which along with the vertical component of synaptic neurotransmission leads to oscillating pattern of neuronal activity. Thirdly, the introduction of inhibitory interneurons into the circuit is expected to equilibrate the circuit operations. Further work is needed to find the region of attraction at the equilibrium point for the resulting nonlinear dynamical system of a given neuronal circuitry at specific time-points. The outputs from the circuit units can be used to provide additional EPSPs to subthreshold activated motor neurons to execute behavioral motor activity. It may become feasible to build independent modules operating in a distributed manner controlling multiple redundant manipulators for generating collective behavioral motor responses as explained in the previous work [60]. The sensations resulting from motor movements are expected to provide feedback inputs to the system.

\section{Conclusion}

The present work has provided a modifiable analogue circuit that can operate similar to the functions of the hypothetical interpostsynaptic functional LINK. These units can be assembled in multiples to build artificial robotic systems with the expectation that the first-person properties of nervous system function will become empirically available along with matching surrogate behavioral motor activities. The expectation of the formation of internal sensation (that cannot be sensed by a third person) concurrent with surrogate motor activity (that can be sensed by a third person) may be compared to the formation of electromagnetism (that cannot be sensed by us) concurrent with the surrogate deflection of a conductor (that can be sensed by us) carrying current in a magnetic field. The circuit features from which the formation of semblances is expected can be replicated using electronic circuits along with in silico software simulations for understanding the rules for the integration of semblances. Scaling up of the present circuit model can lead to the development of robotic systems, which are expected to continuously learn from uncertain inputs from natural scenarios, show behavioral motor activities, and respond to both immediate and delayed rewards. Variations are expected in the pattern of arrangement of the same basic units among the already catalogued 1.12 million animal species along with predicted $9.92 \pm 1.1$ million uncatalogued animal species on earth [61]. Each species has evolved neuronal circuits to make optimal internal sensations of predictions capable of executing the required behavioral motor activity that enables their survival. The large number of animal species increases the probability that trial and error methods of assembly of the electronic circuit units will lead to the generation of systems that mimic one of their nervous systems.

$\begin{array}{ll}\text { Abbreviations } \\ \text { AND: } & \text { AND logic gate } \\ \text { DC: } & \text { Direct current } \\ \text { Dn: } & \text { Dendrite } \\ \text { EPSP: } & \text { Excitatory postsynaptic potential } \\ \text { IC: } & \text { Integrated circuit } \\ \text { IPS: } & \text { Interpostsynaptic location } \\ \text { LINK: } & \text { Interpostsynaptic functional link } \\ \text { LTP: } & \text { Long-term potentiation } \\ \text { mEPSP: } & \text { Miniature excitatory postsynaptic potential } \\ \mathrm{N}_{\mathrm{A}}: & \text { Neuron A } \\ \mathrm{N}_{\mathrm{C}}: & \text { Neuron C } \\ \text { OR: } & \text { OR logic gate } \\ \text { Post: } & \text { Postsynaptic terminal } \\ \text { Postsynapse: } & \text { Postsynaptic terminal (dendritic spine) or } \\ & \text { input terminal } \\ \text { Pre: } & \text { Presynaptic terminal } \\ \text { Presynapse: } & \text { Presynaptic terminal (axonal terminal) or } \\ & \text { output terminal } \\ V_{\text {In }}: & \text { Input voltage } \\ V_{\text {Out }}: & \text { Output voltage. }\end{array}$

\section{Conflict of Interests}

U.S. patent application 14/068,835 pertains to this work.

\section{Acknowledgments}

The author acknowledges the support from Neurosearch Center, Toronto. The author thanks the editor and the reviewers for their helpful comments.

\section{References}

[1] K. Fukushima, Y. Yamaguchi, M. Yasuda, and S. Nagata, "An electronic model of the retina," roceedings of the IEEE, vol. 58, no. 12, pp. 1950-1951, 1970.

[2] C. Koch and B. Mathur, "Neuromorphic vision chips," IEEE Spectrum, vol. 33, no. 5, pp. 38-46, 1996. 
[3] E. D’Angelo, S. Solinas, J. Garrido et al., "Realistic modeling of neurons and networks: towards brain simulation," Functional Neurology, vol. 28, no. 3, pp. 153-166, 2013.

[4] R. Kozma, R. E. Pino, and G. E. Pazienza, Advances in Neuromorphic Memristor Science and Applications, Springer, 2012.

[5] F. Rosenblatt, "The perceptron: a probabilistic model for information storage and organization in the brain," Psychological Review, vol. 65, no. 6, pp. 386-408, 1958.

[6] S. Furber and S. Temple, "Neural systems engineering," Journal of the Royal Society Interface, vol. 4, no. 13, pp. 193-206, 2007.

[7] J. Schmidhuber, "A possibility for implementing curiosity and boredom in model-building neural controllers. In from animals to animats," in Proceedings of the 1st International Conference on Simulation of Adaptive Behavior, pp. 222-227, MIT Press, Cambridge, Mass, USA, 1991.

[8] R. H. Sutton and A. G. Barto, Reinforcement Learning: An Introduction, MIT Press, Cambridge, Mass, USA, 1998.

[9] P.-Y. Oudeyer and F. Kaplan, "What is intrinsic motivation? A typology of computational approaches," Frontiers in Neurorobotics, vol. 1, p. 6, 2007.

[10] M. Minsky, "K-Lines: a theory of memory," Cognitive Science, vol. 4, no. 2, pp. 117-133, 1980.

[11] V. S. Ramachandran and W. Hirstein, "Three laws of qualia: what neurology tells us about the biological functions of consciousness," Journal of Consciousness Studies, vol. 4, no. 5-6, pp. 429-457, 1997.

[12] G. M. Edelman, Bright Air, Brilliant Fire: On the Matter of the Mind, Basic Books, New York, NY, USA, 1992.

[13] F. Crick and C. Koch, "Consciousness and neuroscience," Cerebral Cortex, vol. 8, no. 2, pp. 97-107, 1998.

[14] R. S. Rosenbaum, D. T. Stuss, B. Levine, and E. Tulving, “Theory of mind is independent of episodic memory," Science, vol. 318, no. 5854, article 1257, 2007.

[15] M. Minsky, "Machinery of consciousness," in Proceedings of the 75th Anniversary Symposium on Science in Society, National Research Council of Canada, 1991.

[16] A. Cleeremans, "Consciousness: the radical plasticity thesis," Progress in Brain Research, vol. 168, pp. 19-33, 2007.

[17] K. I. Vadakkan, "Framework of consciousness from semblance of activity at functionally linked postsynaptic membranes," Frontiers in Psychology, vol. 1, article 168, 2010.

[18] B. J. Baars and S. Franklin, "How conscious experience and working memory interact," Trends in Cognitive Sciences, vol. 7, no. 4, pp. 166-172, 2003.

[19] K. I. Vadakkan, Semblance Hypothesis of Memory, iUniverse Publishers, Bloomington, Ind, USA, 3rd edition, 2010.

[20] K. I. Vadakkan, "A supplementary circuit rule-set for the neuronal wiring," Frontiers in Human Neuroscience, vol. 7, article 170, 2013.

[21] L. M. Ward, "Synchronous neural oscillations and cognitive processes," Trends in Cognitive Sciences, vol. 7, no. 12, pp. 553559, 2003.

[22] T. Harmony, "The functional significance of delta oscillations in cognitive processing," Frontiers in Integrative Neuroscience, vol. 7, article 83, 2013.

[23] P. J. Uhlhaas and W. Singer, "High-frequency oscillations and the neurobiology of schizophrenia," Dialogues in Clinical Neuroscience, vol. 15, no. 3, pp. 301-313, 2013.

[24] W. Klimesch, "EEG alpha and theta oscillations reflect cognitive and memory performance: a review and analysis," Brain Research Reviews, vol. 29, no. 2-3, pp. 169-195, 1999.
[25] E. Başar, C. Başar-Eroğlu, S. Karakaş, and M. Schürmann, "Brain oscillations in perception and memory," International Journal of Psychophysiology, vol. 35, no. 2-3, pp. 95-124, 2000.

[26] J. Lisman, "Working memory: the importance of theta and gamma oscillations," Current Biology, vol. 20, no. 11, pp. R490R492, 2010.

[27] M. T. Alkire, A. G. Hudetz, and G. Tononi, "Consciousness and anesthesia," Science, vol. 322, no. 5903, pp. 876-880, 2008.

[28] M. V. Sanchez-Vives and D. A. McCormick, "Cellular and network mechanisms of rhytmic recurrent activity in neocortex," Nature Neuroscience, vol. 3, no. 10, pp. 1027-1034, 2000.

[29] S. G. Hormuzdi, I. Pais, F. E. N. LeBeau et al., "Impaired electrical signaling disrupts gamma frequency oscillations in connexin 36-deficient mice," Neuron, vol. 31, no. 3, pp. 487-495, 2001.

[30] M. R. Deans, J. R. Gibson, C. Sellitto, B. W. Connors, and D. L. Paul, "Synchronous activity of inhibitory networks in neocortex requires electrical synapses containing connexin36," Neuron, vol. 31, no. 3, pp. 477-485, 2001.

[31] A. Mercer, "Electrically coupled excitatory neurones in cortical regions," Brain Research, vol. 1487, pp. 192-197, 2012.

[32] A. Selimbeyoglu and J. Parvizi, "Electrical stimulation of the human brain: perceptual and behavioral phenomena reported in the old and new literature," Frontiers in Human Neuroscience, vol. 4, article 46, 2010.

[33] K. I. Vadakkan, "Processing semblances induced through interpostsynaptic functional LINKs, presumed biological parallels of K-lines proposed for building artificial intelligence," Frontiers in Neuroengineering, vol. 4, article 8, 2011.

[34] G. S. Brindley, "The site of electrical excitation of the human eye," The Journal of Physiology, vol. 127, no. 1, pp. 189-200, 1955.

[35] P. A. Merton and H. B. Morton, "Stimulation of the cerebral cortex in the intact human subject," Nature, vol. 285, no. 5762, p. $227,1980$.

[36] M. S. Beauchamp, P. Sun, S. H. Baum, A. S. Tolias, and D. Yoshor, "Electrocorticography links human temporoparietal junction to visual perception," Nature Neuroscience, vol. 15, no. 7, pp. 957959, 2012.

[37] A. C. Burette, T. Lesperance, J. Crum et al., "Electron tomographic analysis of synaptic ultrastructure," Journal of Comparative Neurology, vol. 520, no. 12, pp. 2697-2711, 2012.

[38] C. A. Anastassiou, R. Perin, H. Markram, and C. Koch, "Ephaptic coupling of cortical neurons," Nature Neuroscience, vol. 14, no. 2, pp. 217-224, 2011.

[39] T. Lomo, "Frequency potentiation of excitatory synaptic activity in the dentate area of the hippocampal formation," Acta Physiologica Scandinavica, vol. 68, supplement 277, p. 128, 1966.

[40] T. V. P. Bliss and T. Lomo, "Long lasting potentiation of synaptic transmission in the dentate area of the anaesthetized rabbit following stimulation of the perforant path," Journal of Physiology, vol. 232, no. 2, pp. 331-356, 1973.

[41] A. Villers and L. Ris, "Improved preparation and preservation of hippocampal mouse slices for a very stable and reproducible recording of long-term potentiation," Journal of Visualized Experiments, no. 76, Article ID e50483, 2013.

[42] D. J. Perkel and R. A. Nicoll, "Evidence for all-or-none regulation of neurotransmitter release: implications for long-term potentiation," Journal of Physiology, vol. 471, pp. 481-500, 1993.

[43] K. Paradiso, W. Wu, and L. G. Wu, "Methods for patch clamp capacitance recordings from the calyx," Journal of Visualized Experiments, no. 6, article 244, 2007. 
[44] P.-A. Buchs and D. Muller, "Induction of long-term potentiation is associated with major ultrastructural changes of activated synapses," Proceedings of the National Academy of Sciences of the United States of America, vol. 93, no. 15, pp. 8040-8045, 1996.

[45] M. Maletic-Savatic, R. Malinow, and K. Svoboda, "Rapid dendritic morphogenesis in CA1 hippocampal dendrites induced by synaptic activity," Science, vol. 283, no. 5409, pp. 1923-1927, 1999.

[46] J. Bourne and K. M. Harris, "Do thin spines learn to be mushroom spines that remember?" Current Opinion in Neurobiology, vol. 17, no. 3, pp. 381-386, 2007.

[47] S. Yagishita, A. Hayashi-Takagi, G. C. Ellis-Davies, H. Urakubo, S. Ishii, and H. Kasai, "A critical time window for dopamine actions on the structural plasticity of dendritic spines," Science, vol. 345, no. 6204, pp. 1616-1620, 2014.

[48] P.-M. Lledo, X. Zhang, T. C. Südhof, R. C. Malenka, and R. A. Nicoll, "Postsynaptic membrane fusion and long-term potentiation," Science, vol. 279, no. 5349, pp. 399-403, 1998.

[49] M. Abeles, Corticonics: Neural Circuits of the Cerebral Cortex, Cambridge University Press, Cambridge, UK, 1991.

[50] L. M. Palmer, A. S. Shai, J. E. Reeve, H. L. Anderson, O. Paulsen, and M. E. Larkum, "NMDA spikes enhance action potential generation during sensory input," Nature Neuroscience, vol. 17, no. 3, pp. 383-390, 2014.

[51] G. J. Rinkus, "A cortical sparse distributed coding model linking mini- and macrocolumn-scale functionality," Frontiers in Neuroanatomy, vol. 4, no. 17, 2010.

[52] J. R. Whitlock, A. J. Heynen, M. G. Shuler, and M. F. Bear, "Learning induces long-term potentiation in the hippocampus," Science, vol. 313, no. 5790, pp. 1093-1097, 2006.

[53] G. C. Baylis, E. T. Rolls, and C. M. Leonard, "Functional subdivisions of the temporal lobe neocortex," Journal of Neuroscience, vol. 7, no. 2, pp. 330-342, 1987.

[54] A. Renart, N. Parga, and E. T. Rolls, "Associative memory properties of multiple cortical modules," Network, vol. 10, no. 3, pp. 237-255, 1999.

[55] H. McGurk and J. MacDonald, "Hearing lips and seeing voices," Nature, vol. 264, no. 5588, pp. 746-748, 1976.

[56] S. Grossberg, "On the serial learning of lists," Mathematical Biosciences, vol. 4, no. 1-2, pp. 201-253, 1969.

[57] S. Grossberg, "Behavioral contrast in short term memory: serial binary memory models or parallel continuous memory models?" Journal of Mathematical Psychology, vol. 17, no. 3, pp. 199-219, 1978.

[58] C. Benkert and D. Z. Anderson, "Controlled competitive dynamics in a photorefractive ring oscillator: winner-takes-all and the voting-paradox dynamics," Physical Review A, vol. 44, no. 7, pp. 4633-4638, 1991.

[59] D. K. Lee, L. Itti, C. Koch, and J. Braun, "Attention activates winner-take-all competition among visual filters," Nature Neuroscience, vol. 2, no. 4, pp. 375-381, 1999.

[60] S. Li, S. Chen, B. Liu, Y. Li, and Y. Liang, "Decentralized kinematic control of a class of collaborative redundant manipulators via recurrent neural networks," Neurocomputing, vol. 91, pp. 1$10,2012$.

[61] C. Mora, D. P. Tittensor, S. Adl, A. G. B. Simpson, and B. Worm, "How many species are there on earth and in the ocean?" PLoS Biology, vol. 9, no. 8, Article ID e1001127, 2011. 

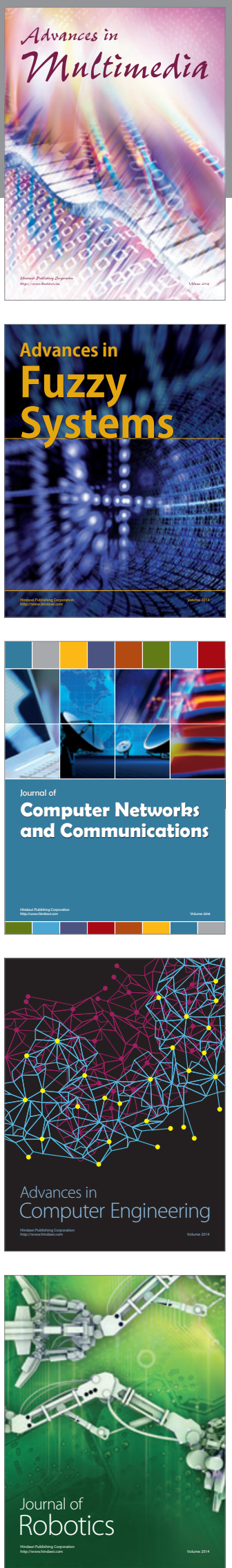

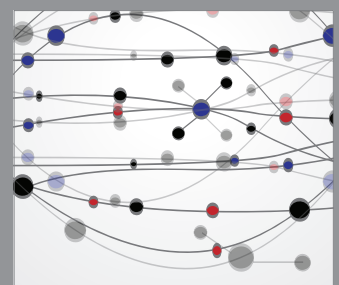

The Scientific World Journal
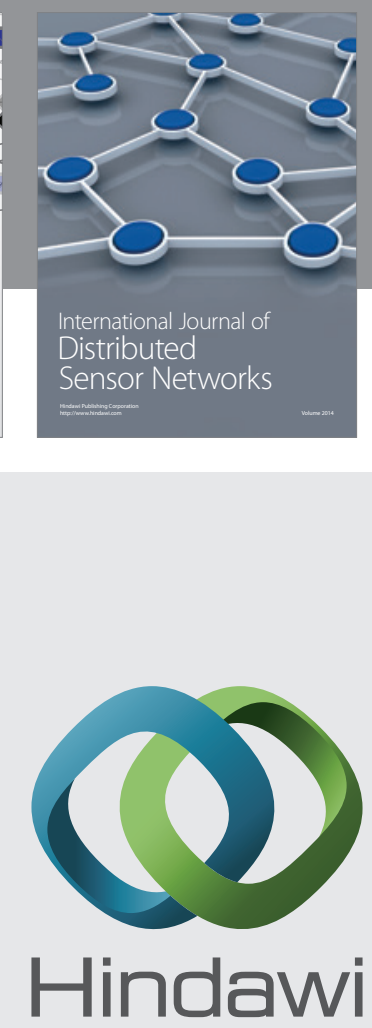

Submit your manuscripts at

http://www.hindawi.com
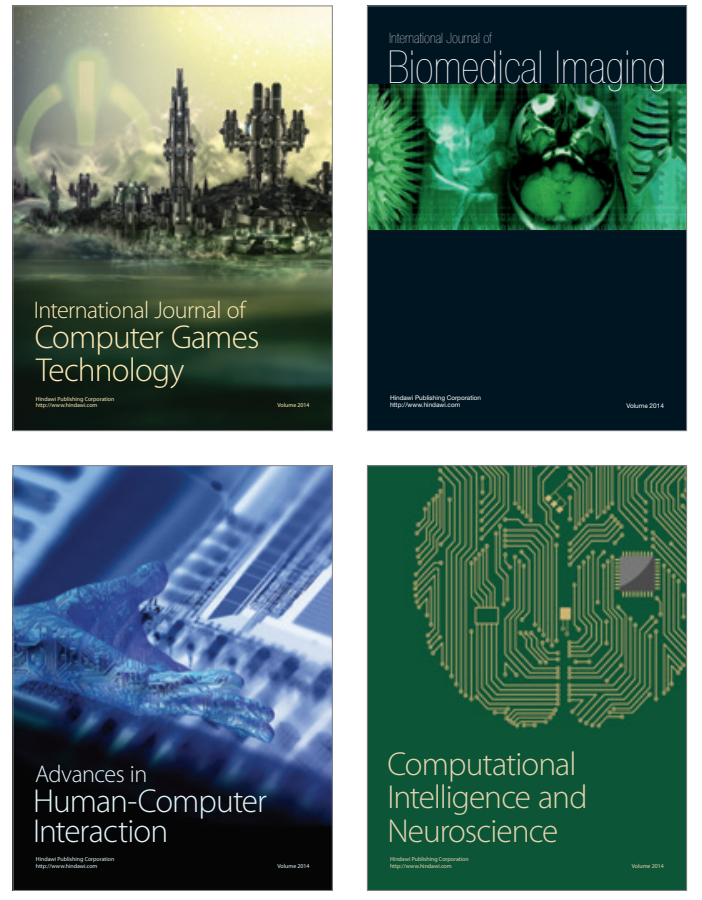
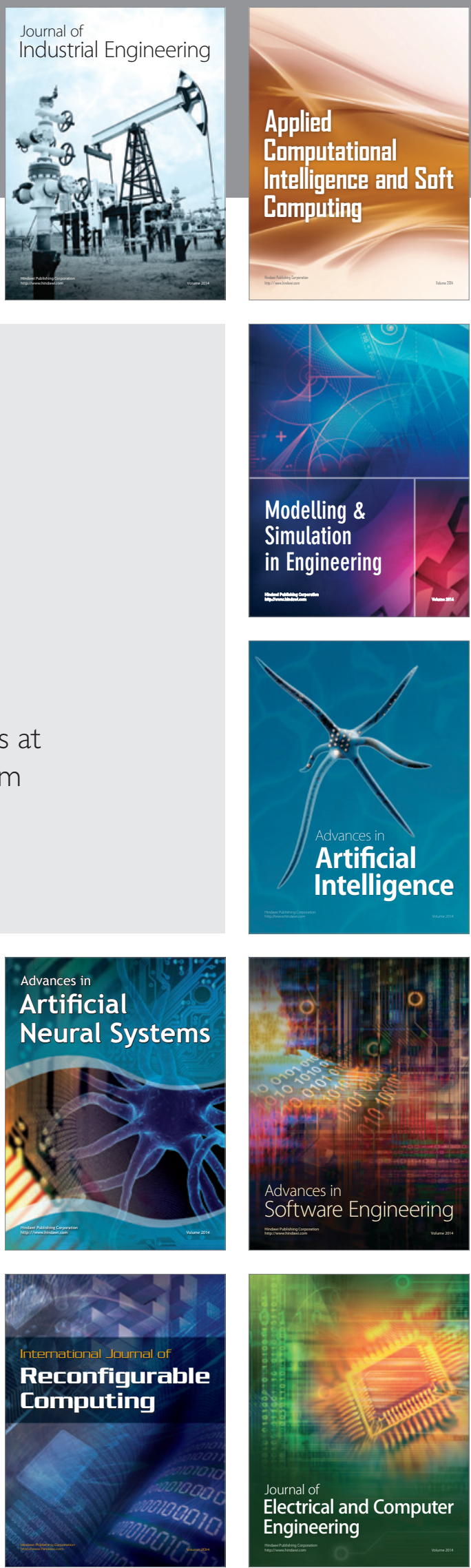